Fuchs, A.-R. \& Bonde, G. J. (1957). J. gen. Microbiol. 16, 317-329

\title{
The Nutritional Requirements of Clostridium perfringens
}

\author{
By ANNA-RIITTA FUCHS AND G. J. BONDE
}

The Institute of Hygiene, University of Copenhagen, Denmark

\begin{abstract}
SUMMARY: The requirements for growth factors, amino acids and inorganic constituents were determined for three strains of Clostridium perfringens (Veillon \& Zuber) Holland. A number of substances were tested as energy sources for this organism, and the influence of $\mathrm{pH}$ value and temperature on growth was determined. The minimal medium evolved contained: alanine, arginine, aspartic acid, cystine, glutamic acid, histidine, isoleucine, leucine (methionine), phenylalanine, threonine, tryptophan, tyrosine and valine; ammonium chloride, magnesium chloride, ferrous chloride, sodium-potassium phosphate buffer; glucose; adenine, biotin, calcium pantothenate and pyridoxine.

For maximal growth the presence of lysine, glycine and serine was necessary. Maximal growth was affected by the balance of amino acids in the medium; the balance of sodium and potassium ions was also important. Certain strain differences were noticed with respect to amino acid and vitamin requirements. Methionine was needed by one strain only; none of the strains required serine (reported essential for strain BP $6 \mathrm{~K}$ of Boyd, Logan \& Tytell, 1948b), whereas aspartic acid was essential for all strains tested but not for BP6 K. Riboflavine, an essential growth factor in BP $6 \mathrm{~K}$, had no effect on growth of the strains tested. One of the strains showed a need for added nicotinamide when transferred to a nicotinamide-free medium after several transfers in peptone water.
\end{abstract}

In an investigation of the sulphur utilization of Clostridium perfringens (Veillon \& Zuber) Holland, a chemically defined medium was needed, and a study of the nutritional requirements was therefore undertaken. Bacteria belonging to the genus Clostridium are usually grown on complex media including beef-infusion broth, glucose + peptone, and tryptic-digested casein, often with added liver or yeast extract. In investigations so far carried out on the nutritional requirements of this group, C. sporogenes, C. tetani and the butyl alcohol-producing clostridia have been given prominence.

The toxin production of Clostridium perfringens has been studied by several investigators and for this purpose many simplified media have been described (Taylor \& Stewart, 1941; Reed \& Orr, 1941; Robertson \& Keppie, 1941; Stewart, 1942; Adams \& Hendee, 1945; Logan, Tytell, Danielson \& Griner, 1945; Tamura, Tytell, Boyd \& Logan, 1941). However, all these media are based upon enzymically digested casein, meat-infusion or peptones with addenda such as meat or liver extracts.

Growth of Clostridium perfringens in a defined medium was first reported by Gladstone, Fildes \& Richardson (1935) who used a mixture of amino acids, inorganic salts, glucose and mercaptoacetic acid, with the addition of a preparation from mare urine (later, of yeast) the 'sporogenes vitamin' of Knight \& Fildes (1933). Shull \& Peterson (1948) showed that the activity of 
'sporogenes vitamin' preparation was probably due to its containing biotin and $p$-aminobenzoic acid. Pappenheimer \& Shaskan (1944), in a study of the role of iron in the carbohydrate metabolism of $C$. perfringens, obtained growth in a casein-hydrolysate Tryptone (Difco) medium and in a partially defined medium based on hydrolysed purified gelatin with added amino acids, glucose, inorganic salts, and known growth factors, but only with regard to iron were the requirements studied more closely. In the course of their investigations on the toxin production of $C$. perfringens, Boyd, Logan \& Tytell (1948a) studied the growth requirements more closely with special reference to vitamin and amino acid requirements, and a defined medium was described.

With our strains certain differences in the amino acid and vitamin requirements as compared with the findings of Boyd et al. were observed. Experiments were therefore carried out on the nutritional requirements of several strains and extended to examine also the inorganic constituents and energy sources. The present paper reports the results of this work on three different strains of Clostridium perfringens.

\section{METHODS}

Organisms. All the strains used were isolated in sulphite glucose iron agar (Wilson \& Blair, 1924) from rotting tank sludge obtained from the Municipal Sewage Treatment Works at Copenhagen. The samples of sludge were pasteurized $\left(80^{\circ}, 5 \mathrm{~min}\right.$.) before incubation, and black colonies which developed were identified by microscopy, fermentation of sugars, and the stormy fermentation reaction in skim milk.

Cultivation. Experiments were carried out in test tubes containing $5 \mathrm{ml}$. medium. After inoculation the tubes were evacuated by means of an oil pump, sealed (see Lebert, 1949) and incubated at $37^{\circ}$ for $20 \mathrm{hr}$. In experiments where the requirements for micronutrients were determined, special test tubes of Pyrex or Jena glass were used and incubated in anaerobic jars. Control experiments showed that growth was identical with both methods.

Growth was estimated by reading optical densities of the cultures with a Lumetron photoelectric colorimeter Model $402 \mathrm{E}$, later by a Beckman colorimeter Model $\mathrm{C}$ with a red filter, with uninoculated culture medium as the blank. The Beckman colorimeter had the advantage that selected Pyrex culture tubes could be used as cuvettes, making it possible to follow anaerobic growth continuously in the same tube. The nitrogen content of a concentrated washed bacterial suspension was determined by the micro-Kjeldahl method, and the optical density of a sample was measured in a series of dilutions. Within the range of optical density of $0 \cdot 14$ to 0.65 scale units the increase in optical density was found to be linear with the nitrogen content. The optical density observed was converted to $\mu \mathrm{g}$. $\mathrm{N} / \mathrm{ml}$. medium by a multiplication constant $k=100 \cdot 8$.

Preparation of inoculum. Stock cultures were grown anaerobically in peptone water and maintained by daily transfers. After incubation for $15 \mathrm{hr}$. the organisms were centrifuged down, washed and resuspended in the buffered salt 
solution used in the preparation of the medium. In experiments on the inorganic requirements, the constituent under investigation was omitted from the wash solution. The nitrogen content of the inocula varied between 5 and $20 \mu \mathrm{g}$. $\mathrm{N} / \mathrm{ml}$, the size of the inoculum within this range being without influence on the amount of growth obtained; as inoculum $0 \cdot 1 \mathrm{ml}$. washed suspension was used per $5 \mathrm{ml}$. medium.

Preparation of media. The composition of the media used is given in Table 1. The substances numbered from 1 to 5 constituted the buffered salt solution used as wash solution.

The $\mathrm{pH}$ value of all media was adjusted to $7 \cdot 0-7 \cdot 2$ with sodium hydroxide except for sodium-free medium where potassium hydroxide was used. The $\mathrm{pH}$ value for routine use was determined with indicator papers (Special Indicators, Merck); in other cases electrometrically (Radiometer). The media with the omission of the constituent under investigation were pipetted into culture tubes and autoclaved at $110^{\circ}$ for $10 \mathrm{~min}$.

All the reagents used were of analytical grade (Merck); the amino acids were obtained from Hoffmann, La Roche and Co., the water used was doubledistilled.

\section{RESULTS}

\section{Effect of inoculum, $\mathrm{pH}$ value and temperature}

Luxuriant growth occurred in both media 1 and 2. Because of the appreciable difference in cost the partially defined medium 2 was used in all preliminary experiments for determining the optimal $\mathrm{pH}$ value, temperature and incubation time, also in preliminary experiments on growth factor requirements and carbon utilization. Medium 1 was used in the final determinations of the basal requirements, in investigations concerning the nitrogen requirements, and with certain modifications in evaluating the need for inorganic constituents. Twice the concentration of sodium and potassium salts was originally used, but when the sodium requirements had been determined, we changed to media 1 and 2.

In determining whether a substance is essential for growth of bacteria two different methods are available: (1) subcultivation in a medium lacking the constituent under examination until growth eventually ceases; (2) washing the bacteria carefully before inoculation in order to remove traces of growth factors and micronutrients which might be carried over with the inoculum. Preliminary experiments indicated that with the latter method reliable results are obtained and much time saved.

Different washing procedures were tried, using sterile distilled water, $0 \cdot 8 \%$ sodium chloride, or the buffered salt solution ( 1 to 5 ) of medium 1 . With unwashed organisms as inoculum growth could be observed by naked eye $4 \mathrm{hr}$. after inoculation, and maximum growth was attained within $10 \mathrm{hr}$. When physiological saline was used for washing the lag period was prolonged, growth not being observable in the $11 \mathrm{hr}$. following inoculation, maximum growth nevertheless being obtained within $20 \mathrm{hr}$. With buffered salt solution as washing medium growth was retarded as compared with unwashed organisms, but in this case growth was evident within $6-8 \mathrm{hr}$; $10 \mathrm{hr}$. after inoculation almost 


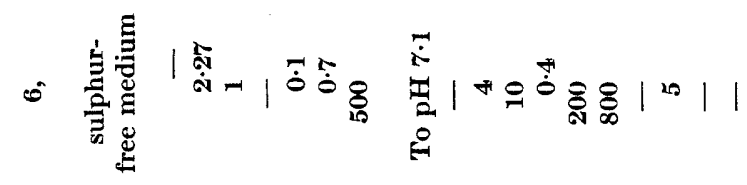

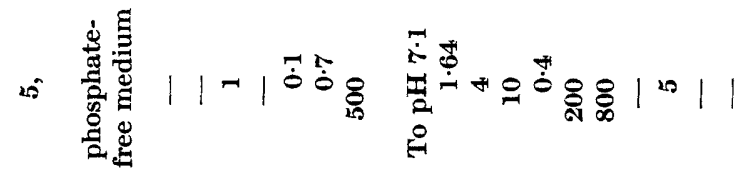

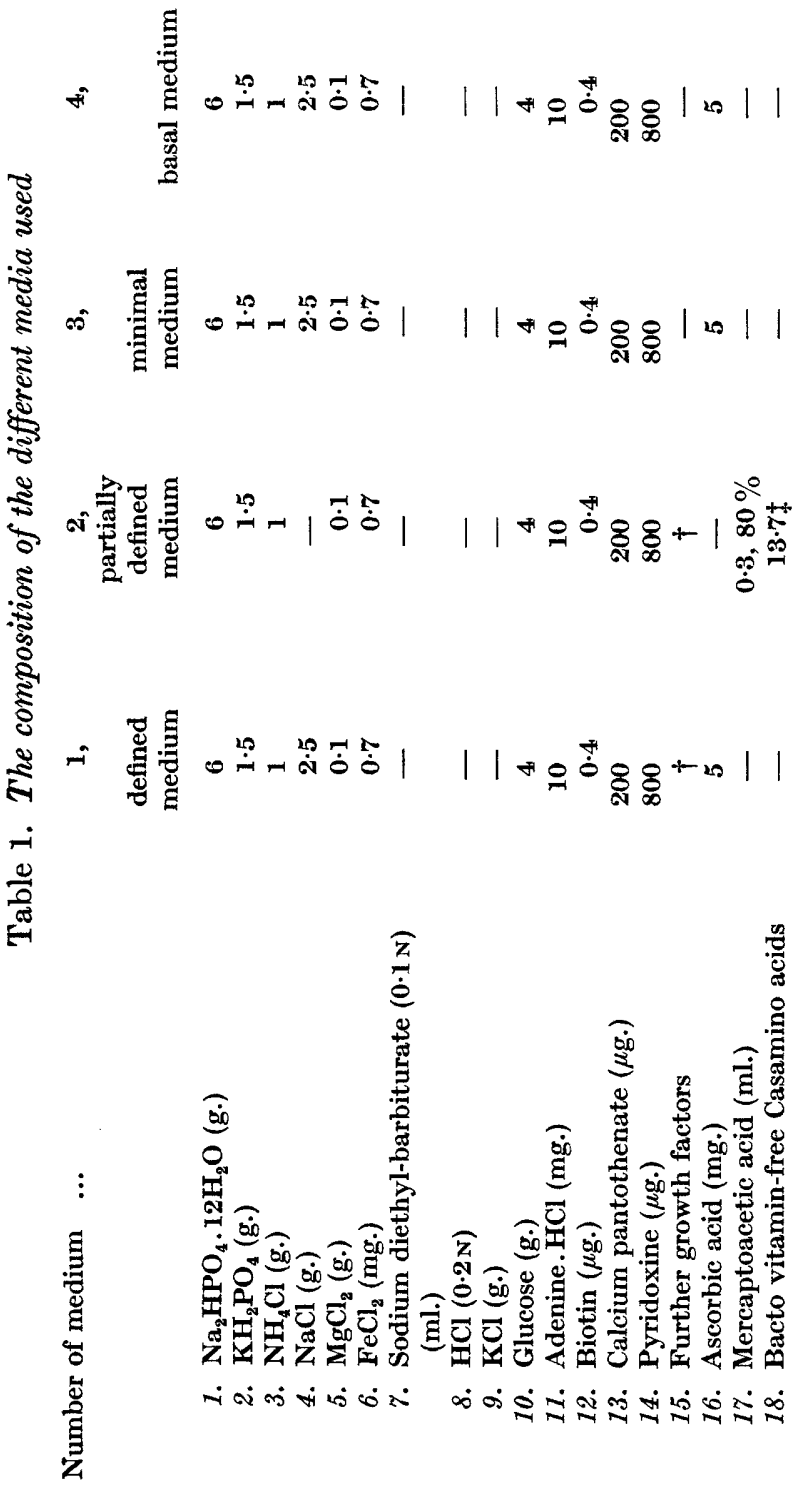




$=111111111111111111$
$=111111111111111111$

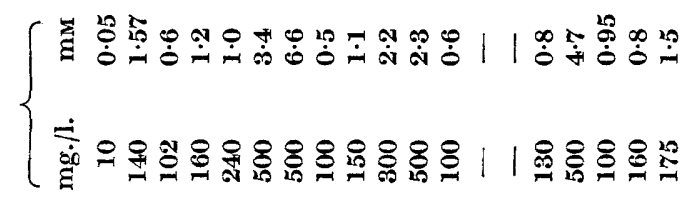

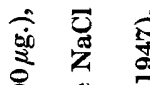

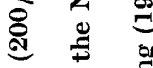

률

:

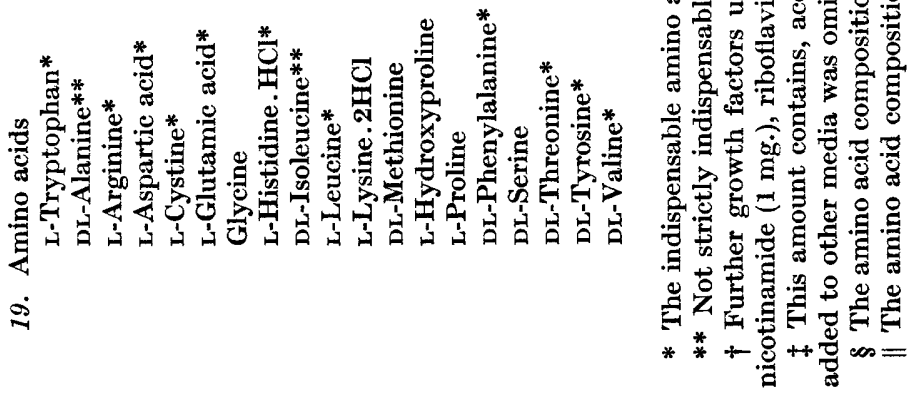


maximal growth was obtained. When the organisms were washed with distilled water the results were variable and often only slight growth occurred.

Addition of reducing agents (e.g. mercaptoacetic acid) in low concentrations to the wash solutions did not improve the growth rate obtained; with higher concentrations a marked prolongation of the lag phase was observed.

The rate of growth also markedly depended on the age of the culture, whereas the number of bacteria in the inoculum, within the range used (equivalent to $2-20 \mu \mathrm{g} . \mathrm{N} / \mathrm{ml}$.) was without influence on the maximal growth. With a $4 \mathrm{hr}$. culture as inoculum, washed in the buffered salt solution, maximum growth was obtained within $6 \mathrm{hr}$.; with a $15 \mathrm{hr}$. culture growth became evident within $6 \mathrm{hr}$., but the maximum growth was not reached until 10-18 hr.; with a $20 \mathrm{hr}$. culture growth did not begin before $10 \mathrm{hr}$., with maximal growth within $20 \mathrm{hr}$.

In the defined medium autolysis started soon after $25 \mathrm{hr}$. of incubation, the turbidity decreasing rapidly.

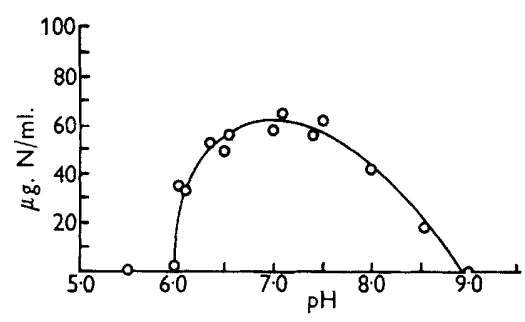

Fig. 1. The effect of $\mathrm{pH}$ upon the growth of Clostridium perfringens.

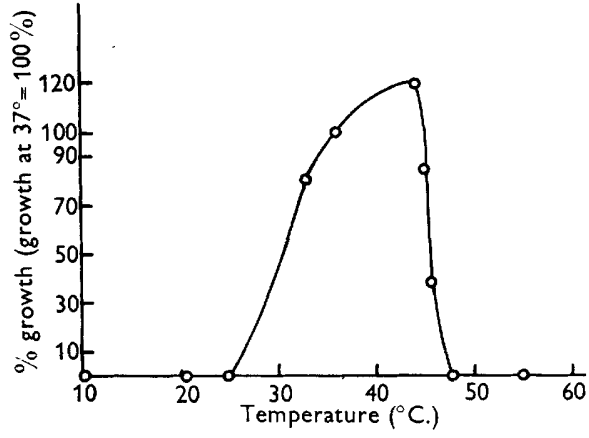

Fig. 2. The dependence of the growth of Clostridium perfringens on temperature (Growth at $37^{\circ}=100 \%$.)

Boyd et al. (1948a) reported an acceleration of growth by adding adenine, guanine or uracil, maximum growth occurring also in a medium without added purines, although at a slow rate (within $25 \mathrm{hr}$.). The strains tested in the present work showed an absolute requirement for adenine, not even retarded growth occurring in its absence or when it was replaced by the other purines.

Gladstone et al. (1935) stated that carbon dioxide is an essential factor for certain bacteria including Clostridium perfringens, and that carbon dioxide must be present in all media before multiplication begins. In the present work added carbon dioxide was not necessary for the growth of the $C$. perfringens strains used. In all cases maximal growth was obtained within $20 \mathrm{hr}$.

The optimal $\mathrm{pH}$ value of the medium was determined, the relationship between growth and $\mathrm{pH}$ value being shown in Fig. 1; optimal growth was obtained between $\mathrm{pH} \mathbf{6 \cdot 7 5}$ and $\mathbf{7 \cdot 5}$.

The growth-temperature relationship was determined for a strain which had been maintained in laboratory media at $37^{\circ}$ for 10 weeks. The tubes were incubated at ten different temperatures in medium 2 . The results are shown in Fig. 2; optimal growth occurred between $37^{\circ}$ and $44^{\circ}$. 
Neither medium 1 nor 2, which include the growth factors, supported growth unless a reducing agent was added; mercaptoacetic acid, cysteine or ascorbic acid were effective; thioacetamide and sulphide were ineffective. Better growth was obtained with mercaptoacetic acid than with ascorbic acid; except when a sulphur-free medium was desired, mercaptoacetic acid was generally used.

Vitamin requirements

No growth occurred in medium 1 or 2 with added ferrous ions and mercaptoacetic acid when the other accessory factors were omitted, but the addition of a group of known growth factors, as listed in Table 1, resulted in heavy growth. The accessory factors were then individually omitted from the medium in a series of experiments.

The following were found to be necessary for the growth of the strains of Clostridium perfringens tested (concentrations for optimal growth in brackets; $\mu \mathrm{g} . / \mathrm{ml}$.): adenine $(10)$; calcium pantothenate $(0 \cdot 2)$; pyridoxine $(0 \cdot 8)$; biotin $(0 \cdot 00004)$.

The growth obtained in medium 1 or 2 in the presence of these growth factors was as heavy as that obtained in meat extract broth + ferrous ions + mercaptoacetic acid or in peptone + glucose. Riboflavine (reported to be essential for strain BP $6 \mathrm{~K}$ by Boyd et al. 1948 $a, b$ ), did not have any effect on the growth of the strains tested, neither did the addition of other growth factors have any stimulatory effect. The growth response to adenine, calcium pantothenate, pyridoxine and biotin was similar to that described by Boyd et al. Adenine, calcium pantothenate and pyridoxine were indispensable for all strains studied. Some growth occurred in a medium lacking added biotin, but the addition of biotin was then greatly stimulating. Some lots of the Bacto Vitamin-free Casamino acids contain traces of adenine or some substance equivalent to adenine, occasionally also traces of B-vitamins and tryptophan, because some growth occurred even without the addition of these factors, although the same culture in the completely defined medium 1 did not grow unless these substances were added.

Nicotinic acid or nicotinamide was not required by freshly isolated strains and did not have any stimulatory effect, but after many passages in laboratory media rich in nicotinic acid (peptone water) some strains grow much better when nicotinamide or nicotinic acid was added to a nicotinamide-free medium. These strains also lost their sulphite-reducing capacity in a medium which lacked nicotinamide.

\section{Inorganic requirements}

Following the determination of the basal physical and chemical requirements of the culture medium the effect of the balance of the chemicals used was investigated.

Sodium and potassium. The effect of sodium and potassium ions in varying concentrations was studied. The sodium-free medium consisted of the minimal medium, number 3 in Table 1, but with the sodium-potassium phosphate buffer and the sodium chloride replaced by a buffer prepared with primary 
potassium phosphate, potassium hydroxide and potassium chloride; sodium was added as sodium phosphate. The potassium-free medium was prepared in a similar manner with a pure sodium phosphate buffer and sodium chloride; potassium was added as chloride. No special precautions were taken to remove traces of the two alkali metals present as impurities.

Table 2. The dependence of growth of Clostridium perfringens on sodium and potassium concentrations

A. Increasing sodium concentration with constant potassium concentration at different potassium concentrations.

$\begin{array}{cccc}\begin{array}{c}\text { Normality } \\ \text { of Na }\end{array} & \mathbf{K}=\mathbf{0 . 0 5 N} & \mathbf{K}=\mathbf{0 . 1 2 5 N} & \mathbf{K}=\mathbf{0 . 2 1 5 \mathrm { N }} \\ \mathbf{0} & \div & \div & \div \\ \mathbf{0} .0001 & \div & \div & \div \\ \mathbf{0} \cdot 001 & \div & \div & \div \\ 0.003 & + & + & \div \\ 0.01 & ++ & ++ & \div \\ \mathbf{0} \cdot 1 & ++ & + & + \\ 0.25 & \div & \div & + \\ \mathbf{0 . 4 5} & \div & + & + \\ 0.83 & \div & + & +\end{array}$

B. Increasing potassium concentration.

Normality

$\begin{array}{lcc}\text { of } \mathrm{K} & \mathrm{Na}=0.119 \mathrm{~N} & \mathrm{Na}=0.166 \mathrm{~N} \\ 0 & (+) & (+) \\ 0.0001 & ++ & ++ \\ 0.0004 & ++ & ++ \\ 0.001 & ++ & + \\ 0.01 & ++ & + \\ 0.1 & + & (+)\end{array}$

$\div$ denotes no growth; $(+),+,++$ denotes increasing degrees of growth.

It is seen from the results (Table 2) that in their influence upon the growth of Clostridium perfringens in a defined medium, the concentrations of sodium and potassium ions were mutually dependent. Sodium was indispensable, but only traces of potassium were needed, some growth occurring without added potassium. Additional potassium, however, enhanced growth, and optimal growth was obtained with potassium concentrations varying from 0.0001 to $0.05 \mathrm{M}$; higher potassium concentrations depressed growth unless the sodium concentration was also increased. The threshold concentration of sodium changed from 0.03 to $0.1 \mathrm{M}$ when the potassium concentration was increased from 0.05 to $0.215 \mathrm{M}$. On the other hand, growth was possible with potassium at $0 \cdot 1-$ $0.215 \mathrm{M}$ with such sodium concentrations as would inhibit growth at lower potassium concentrations. The toxic concentration of sodium was thus raised from 0.25 to $>0.45 \mathrm{M}$ when the potassium was increased from 0.05 to $0.215 \mathrm{M}$. The optimal sodium concentration in the defined minimal medium (number 3 of Table 1 ) varied between 0.01 and $0.1 \mathrm{M}$, depending on the potassium level.

Phosphate. In experiments in which the requirement for phosphate was studied, a sodium diethylbarbiturate buffer was used (medium 5, Table 1). The $\mathrm{pH}$ value was adjusted with $0 \cdot 2 \mathrm{~N}$-hydrochloric acid. Phosphate was 
indispensable for the growth of Clostridium perfringens; optimal growth occurred even with $0 \cdot 00005 \mathrm{M}$-phosphate and the growth remained unaltered up to $0 \cdot 125 \mathrm{M}$, after which it rapidly decreased.

Sulphate. It was not necessary to add either sulphate or any other compound to any of the media 1-4 (with mercaptoacetic acid replaced by ascorbic acid) for the growth of Clostridium perfringens. The question of the availability of sulphur is dealt with in another paper (Fuchs \& Bonde, 1957).

Magnesium. Omission of magnesium chloride from the medium 1 or 2 resulted in complete lack of growth; this was also observed by Webb (1951) who obtained growth even with $1 \mathrm{mg}$./l.; optimal growth was obtained with our strains at 50-100 mg. $\mathrm{MgCl}_{2} / \mathrm{l}$, the same concentration required for normal rod formation according to Webb; concentrations $>100 \mathrm{mg}$./l. did not further increase growth.

Iron. Growth did not occur in a medium completely lacking in iron, provided that the inoculum organisms were well washed. Pappenheimer \& Shaskan (1944) reported maximum growth at $0 \cdot 6 \mathrm{mg}$. ferrous ion/l. medium. This was also the case with our strains; growth was not affected by increases beyond $0 \cdot 6$ up to $90 \mathrm{mg}$./l.

Other elements. When sodium and potassium phosphate, ammonium chloride, magnesium chloride and ferrous chloride were present in optimal amounts, the addition of other inorganic nutrients was superfluous. No increase in growth occurred when manganese, copper, zinc, and calcium were added singly or together.

\section{Nitrogen requirements}

The nitrogen required by Clostridium perfringens can be supplied by acidhydrolysed casein, provided that L- or DL-tryptophan and an ammonium salt are added. The casein hydrolysate may be substituted by a mixture of 19 pure amino acids of approximately the same composition as $1 \%$ casein (based on the data of Block \& Bolling, 1947), the growth being then as heavy as with casein hydrolysate (amino acids used listed Table 1). The concentration of the amino acids could be decreased to one-fourth without affecting growth.

Ammonium ion cannot be omitted from the medium; without added ammonia slight growth occurred in the defined medium 1 , and some in medium 2 which probably contained traces of ammonia; $500 \mathrm{mg}$. ammonium chloride/l. is required for maximal growth.

\section{Amino acid requirements}

When the need for individual amino acids was investigated, some differences in the requirements for different strains were observed. It became evident that the balance of certain amino acids in the medium was of importance. By omission of amino acids one at a time and in groups from the medium 1 , the following were found to be essential for all strains of Clostridium perfringens tested (growth was not observed in their absence): arginine, aspartic acid, cystine, glutamic acid, histidine, leucine, phenylalanine, threonine, tryptophan, tyrosine and valine. Cystine could partly be replaced by mercaptoacetic acid, 
which could supply some of the sulphur needed for growth, but growth was much heavier with cystine.

The effect of methionine varied considerably. The omission of methionine had no effect with strain B, slightly depressed the growth of strain $\mathbf{A}$, and resulted in very poor growth of strain $\mathbf{C}$. The omission of isoleucine and alanine singly from medium $\mathbf{1}$ also resulted in poor growth except with strain $\mathbf{B}$, with which growth was only depressed to some degree in an otherwise complete medium free from isoleucine. In the absence of both no growth occurred.

The essential amino acids, including methionine for the methioninerequiring strain, alanine and isoleucine, constitute the minimal medium for Clostridium perfringens, denoted by 3 in Table 1 together with the buffered salt solution of medium 1 , glucose $(4 \mathrm{~g} . / 1$.$) , ascorbic acid (5 \mathrm{mg} . / 1$.$) , adenine$ (10 mg./l.), biotin (0.04 $\mu \mathrm{g} . / \mathrm{l}$.$) , calcium pantothenate (200 \mu \mathrm{g} . / \mathrm{l}$.$) , and pyri-$ doxine $(800 \mu \mathrm{g} . / 1$.$) . The proportions of the amino acids are important for$

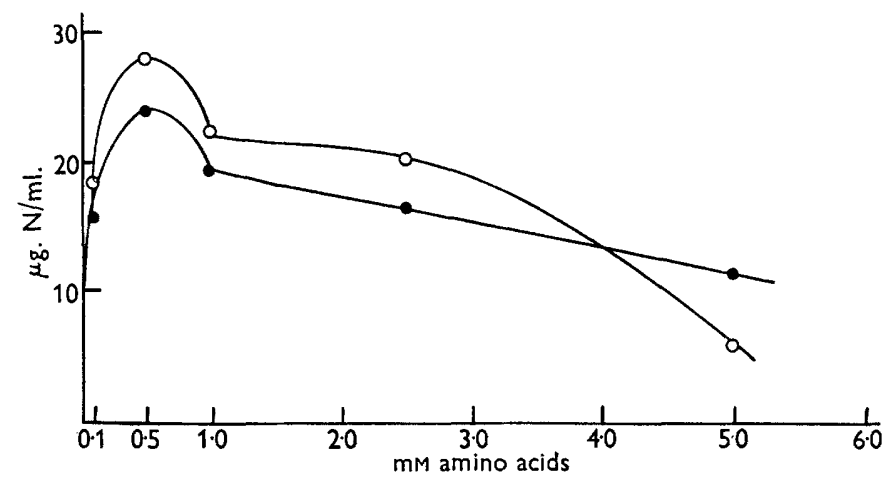

Fig. 3. The effect of increasing concentrations of amino acids on growth of Clostridium perfringens. The individual amino acids are present in equimolar concentrations, except L-tryptophan, which is only $0.05 \mathrm{~mm}$. O : indispensable amino acids; $O$ : indispensable amino acids + methionine.

maximal growth. In the mixture which approximates to $1 \%$ casein, where the acids are present in concentrations varying from $0.17 \mathrm{~mm}$ (cystine) to $15.5 \mathrm{~mm}$ (glutamic acid), the growth response was very good. When the acids were added in equimolar concentrations varying from 0.1 to $5.0 \mathrm{~mm}$ the growth response was maximal at $0.5 \mathrm{mu}$ (Fig. 3); only half maximal growth occurred in the $5 \cdot 0 \mathrm{~mm}$ medium.

The minimal amounts of amino acids which supported maximal growth in the complete medium of Boyd et al. (1948b) for strain BP $6 \mathrm{~K}$ were the following (calculated from the data of Boyd et al., expressed in mmole/1.): L-arginine, 0.25 ; L-cystine, 0.083 ; L-glutamic acid, ${ }_{4} 0.81$; L-histidine, 0.096 ; DL-isoleucine, 0.92 ; L-leucine, 0.38 ; DL-phenylalanine, 0.48 ; DL-threonine, 0.67 ; L-tryptophan, 0.39 ; L-tyrosine, $0 \cdot 19$; DL-valine, $0 \cdot 13$.

The minimal medium 3 of Table 1 supported growth of the three strains tested, well with strain A, rather poorly with strain C. Better growth in all cases was obtained in a medium which contained the indispensable amino 
acids alanine, isoleucine and methionine in concentrations corresponding to $\mathbf{0 . 2 5} \%$ casein, the stimulatory amino acids glycine, serine and lysine being present in the high concentrations of Boyd et al. $(1948 b)$. This is the basal medium, number 4 in Table 1.

Antagonism between amino acids. Interrelationships between the remaining amino acids were noticed, indicating the existence of antagonistic effects similar to those observed with other organisms (Gladstone, 1939; Bonner, 1946; Doermann, 1944; Rowley, 1953; Washburn \& Niven, 1948). In the case of Clostridium perfringens, however, the inhibitions did not seem to be specific, the action of certain amino acids being prevented by several other amino acids. The' degree of inhibition varied also in the different strains, being most marked in strain C. Two groups were involved; glycine, lysine and serine, and hydroxyproline and proline.

In the minimal medium, number 3 in Table 1, the addition of glycine, lysine or serine improved growth, glycine having the greatest effect and lysine the smallest; hydroxyproline and proline had no effect. When added together, glycine + lysine had a synergistic effect; added serine depressed both the glycine and lysine effects. Hydroxyproline and proline were mutually dependent. With the medium containing the complete mixture of amino acids present in casein, the removal of proline resulted in considerable improvement in growth; following the omission of hydroxyproline growth remained unaltered, but the simultaneous omission of both depressed the growth obtained. The concentration of glycine, however, was low in the medium $1(0 \cdot 65 \mathrm{mmole} / \mathrm{l}$.$) ;$ when the concentration was increased to $6 \cdot 6$ mmole/l. the growth remained unaffected by the removal of the proline group from the medium. Maximal growth thus occurred in the basal medium, where the glycine concentration was high (6.6 mmole/l.) in the absence of the proline group.

The amino acid requirements determined by Boyd et al. for Clostridium perfringens strain $\mathrm{BP} 6 \mathrm{~K}$ differ in some respects from those found in the present work. As with our strains, arginine, cystine, glutamic acid, histidine, leucine, phenylalanine, threonine, tyrosine, valine and tryptophan were found to be essential, but also isoleucine, methionine and serine were absolute requirements of strain $\mathrm{BP} 6 \mathrm{~K}$, whereas alanine and aspartic acid were not. However, they found that pyridoxamine or pyridoxal (or pyridoxine in amounts a thousand-fold larger) apparently eliminated the requirement of strain BP6 K for lysine, alanine, aspartic acid and glycine. In the presence of pyridoxine in amounts which supported growth in the otherwise complete medium alanine and lysine were essential, and only very poor growth occurred in the absence of glycine or aspartic acid. The minimal amounts which supported maximal growth of $\mathrm{BP} 6 \mathrm{~K}$ in the otherwise complete medium were given in another paper by Boyd et al. (1948b). Only in the case of L-tryptophan was the optimal concentration determined in the present work. As shown in Fig. 4, $2 \cdot 25 \mathrm{mg}$. L-tryptophan/l. were required by our strain $\mathrm{A}$ in medium 2 to obtain maximal growth, which is considerably lower than the concentration required for $\mathrm{BP} 6 \mathrm{~K}$ (8.0 mg./l.). 


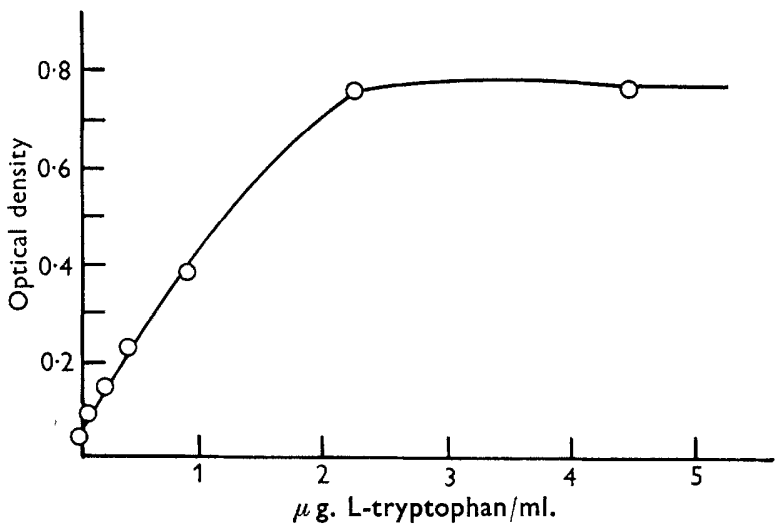

Fig. 4. Growth response of Clostridium perfringens to L-tryptophan in medium 2. Growth remains unaltered by increasing concentration until $180 \mu \mathrm{g} . / \mathrm{ml}$. At $450 \mu \mathrm{g} . / \mathrm{ml}$. the O.D. is 0.35 scale units.

\section{Utilization of carbon compounds}

Clostridium perfringens is strictly dependent on carbohydrates and similar compounds for its energy; no growth occurred in the casein hydrolysate medium 2 without addition of glucose. Other carbon compounds were tested as energy sources; only saccharides were utilized (see Table 3) and only galac-

Table 3. Growth of Clostridium perfringens in defined medium with different carbon compounds as substrates

Growth is expressed in percentage of the growth obtained with an equimolar amount of glucose.

\begin{tabular}{|c|c|c|c|}
\hline Substrate & $\begin{array}{c}\text { Growth } \\
(\%)\end{array}$ & Substrate & $\begin{array}{c}\text { Growth } \\
(\%)\end{array}$ \\
\hline $\begin{array}{l}\text { Polysaccharides } \\
\text { Inulin } \\
\text { Soluble starch }\end{array}$ & $\begin{array}{l}12 \cdot 6 \\
72 \cdot 4\end{array}$ & $\begin{array}{l}\text { Hexoses } \\
\text { Glucose } \\
\text { Galactose }\end{array}$ & $\begin{array}{l}100 \\
104\end{array}$ \\
\hline $\begin{array}{l}\text { Disacchariđes } \\
\text { Saccharose } \\
\text { Maltose } \\
\text { Lactose }\end{array}$ & $\begin{array}{l}88 \cdot 5 \\
72 \cdot 4 \\
66 \cdot 0\end{array}$ & $\begin{array}{c}\text { Fructose } \\
\text { Pentoses } \\
\text { Xylose } \\
\text { Arabinose } \\
\text { Polyalcohol } \\
\text { Mannitol }\end{array}$ & $\begin{array}{r}71 \cdot 0 \\
20 \cdot 6 \\
5 \cdot 3 \\
0 \cdot 6\end{array}$ \\
\hline
\end{tabular}

The following compounds gave no growth: formate, acetate, propionate, butyrate, citrate, succinate, fumarate, pyruvate, lactate, gluconate, tartrate, glycerol, acetone, ethanol. Glycollate gave $7 \%$ and glycerophosphate $5 \%$ of growth in the units used.

tose gave as good growth as glucose. Sodium glycerophosphate, sodium glycollate and glycerol gave only slight growth. The results are summarized in Table 3. Sodium pyruvate had a growth-stimulating effect in the presence of glucose, although it alone did not support growth. Some other substances tested had an inhibitory effect in the presence of glucose; these were (in order of decreasing effectiveness): citrate, acetate, gluconate, succinate and formate.

Our sincere thanks are due to Professor P. Bonnevie, Director of this Institute, for valuable support and encouragement. 


\section{REFERENCES}

Adams, M. H. \& Hendee, E. D. (1945). Methods for the production of the $\alpha$ - and $\theta$-toxin of Clostridium welchii. J. immunol. 51, 249.

Block, R. J. \& Bolling, D. (1947). The Amino Acid Composition of Proteins and Foods. Springfield, Illinois, U.S.A.: Charles C. Thomas.

Bonner, D. (1946). Further studies of mutant strains of Neurospora requiring isoleucine and valine. J. biol. Chem. 166, 545 .

Boyd, M. J., Logan, M. A. \& Tytell, A. A. (1948a). The growth requirements of Clostridium perfringens. J. biol. Chem. 174, 1013.

Boyd, M. J., Logan, M. A. \& Tytell, A. A. (1948b). A microbiological procedure for the assay of amino acids with Clostridium perfringens (welchii) BP6K. J. biol. Chem. 174, 1027.

Doermann, A. H. (1944). A lysineless mutant of Neurospora and its inhibition by arginine. Arch. Biochem. 5, 373.

Fuchs, A.-R. \& Bonde, G. J. (1957). The availability of sulphur for Clostridium perfringens and an examination of hydrogen sulphide production. $J$. gen. Microbiol. 16, 330.

Gladstone, G. P. (1939). Interrelationship between amino acids in the nutrition of B. anthracis. Brit. J. exp. Path. 20, 189.

Gladstone, G. P., Fildes, P. \& Richardson, G. M. (1935). Carbon dioxide as an essential factor in the growth of bacteria. Brit. J. exp. Path. 16, 335.

KNight, B. C. J. G. \& Fildes, P. (1933). A vitamin necessary for the growth of B. sporogenes: its relation to auxin and other growth factors. Brit. J. exp. Path. 14, 112.

LEBert, F. (1949). Technique actuelle d'isolement et de détermination des bactéries anaérobies. Paris: 'Pachomy', Sarl Éditeurs.

Logan, M. A., Tytell, A. A., Danielson, I. S. \& Griner, A. M. (1945). Production of Clostridium perfringens $\alpha$-toxin. J. Immunol. 51, 317.

Pappenheimer, Jun., A. M. \& Shaskan, E. (1944). Effect of iron on carbohydrate metabolism of Clostridium welchii. J. biol. Chem. 155, 265.

ReED, G. B. \& OrR, J. H. (1941). Gas gangrene toxin production in gelatin-thioglycollate medium. Proc. Soc. exp. Biol., N.Y., 48, 535.

Robertson, M. \& Keppie, J. (1941). In vitro production of toxin from strains of Cl. welchii recently isolated from war wounds and air raid casualties. J. Path. Bact. 53, 95.

Rowley, D. (1953). Interrelationships between amino-acids in the growth of coliform organisms. J. gen. Microbiol. 9, 37.

Shull, G. M. \& Peterson, W. H. (1948). The nature of the "sporogenes vitamin" and other factors in the nutrition of Clostridium sporogenes. Arch. Biochem.18, 69.

Stewart, S. E. (1942). Antigenic value of $\mathrm{Cl}$. perfringens ( $\mathrm{Cl}$. welchii) toxoid in prevention of gas gangrene. War Med., Chicago, 2, 87.

Tamura, T. T., Tytell, A. A., Boyd, M. J. \& Logan, M. A. (1941). Production of Clostridium welchii toxin in peptone-free medium. Proc. Soc. exp. Biol., N.Y. 47, 284 .

Taylor, A. W. \& Stewart, J. (1941). The toxins produced by $\mathrm{Cl}$. welchii in a simple medium. J. Path. Bact. 53, 87.

Washburn, M. R. \& Niven, C. F. (1948). Amino acid interrelationships in the nutrition of Streptococcus bovis. J. Bact. 55, 769 .

WEBB, M. (1951). The influence of magnesium on cell division. 5. The effect of magnesium on the growth of bacteria in chemically-defined media of varying complexity. J. gen. Microbiol. 5, 485.

Wilson, W. J. \& BLAIR, E. M. Mc. V. (1924). The application of a sulphite glucose iron agar medium to the quantitative estimation of $B$. welchii and other reducing bacteria in water supplies. J. Path. Bact. 27, 119.

(Received 9 August 1956) 